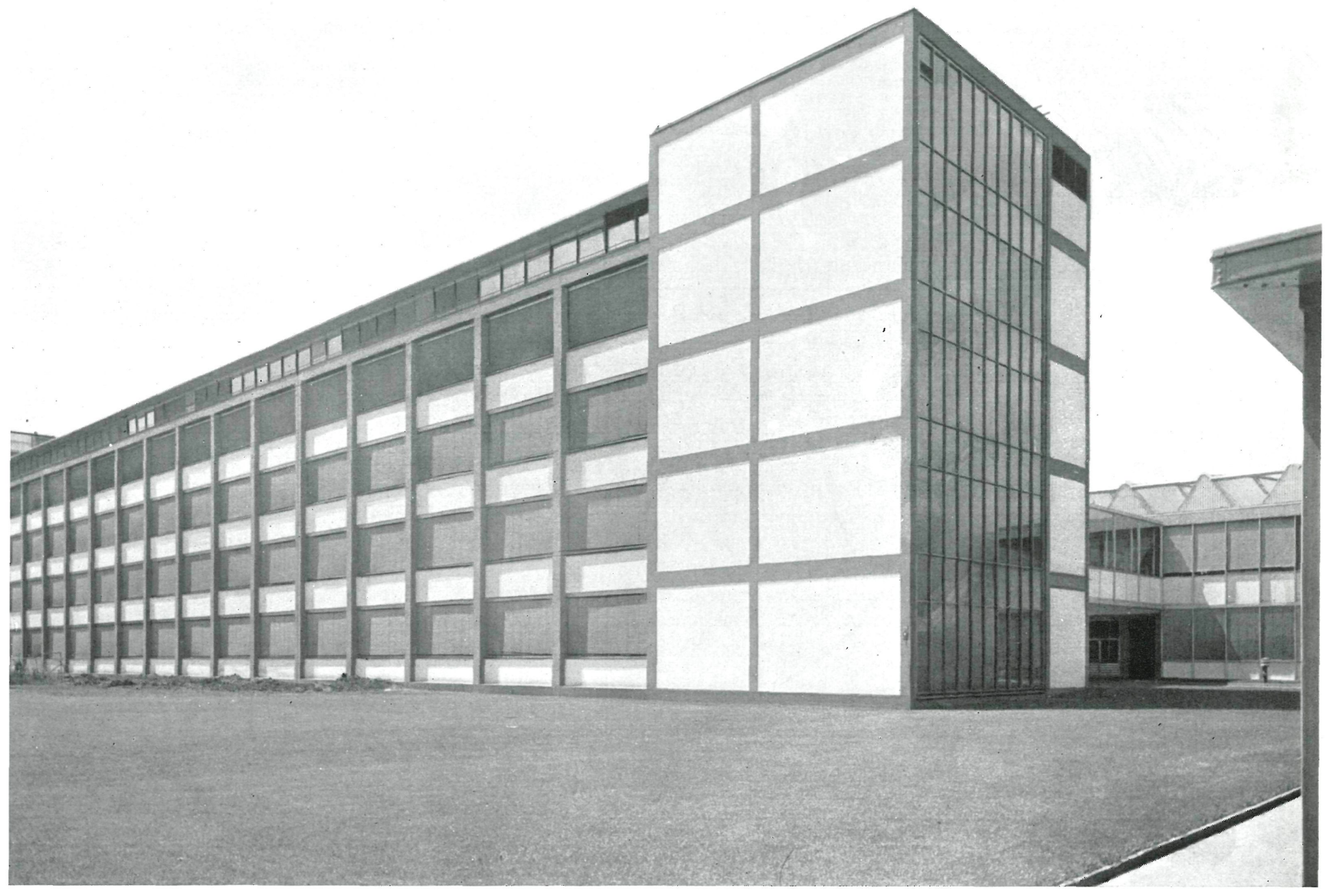

\title{
la oscilación en estructuras de hormigón pretensado y de plantas múltiples
}

H. KUPFER, Dr. ingeniero

simopsis El proyecto para la construcción de un edificio industrial dedicado a la fabricación de mecanismos de precisión se realizó teniendo
en cuenta la influencia de las oscilaciones en la estructura, efecto que, dada la precisión de la mecánica que debía fabricarse, presentaba un problema de notable importancia. Todo el dispositivo estructural se resolvió de acuerdo con estas directrices, y de tal forma que pudieran compararse las previsiones analíticas con ensayos directos obtenidos en el propio edificio.

El edificio tiene $66 \times 15,4 \mathrm{~m}$ en planta y consta de: sótano, entresuelo, cuatro plantas normales, una sexta planta retranqueada unos $3 \mathrm{~m}$ respecto de la fachada $y$ cubierta con un entramado metálico. Como la construcción se ha levantado utilizando piezas prefabricadas, debia tenerse especial cuidado en la termide cipio en una solución a base de pórticos, de $15 \mathrm{~m}$ de luz pretensado. La parte central de los forjados en las distintas plantas la constituye un elemento de sección hueca, utilizado para alojar los conductos del aire acondicionado.

Como la luz de $15 \mathrm{~m}$ de las vigas pretensadas podía influir notablemente en el sistema vibrante, se previeron una serie de mon. tantes, independientes, que podrían montarse y desmontarse fácilmente y que, colocados en la parte central de cada pórtico, pro. porcionarian una especie de apuntalamiento general cuya influencia sería notable. 
En el programa de ensayos se tuvieron en cuenta distintas hipótesis y dispositivos de excitación ondulatoria y dispositivos de registro en oscilógrafos.

El autor se extiende, con gran detalle, en los distintos ensayos realizados y en los métodos utilizados para recoger los datos que permitirían un análisis general del sistema vibrante, sus modificaciones generales y resonancias, amplitudes y onda propia según los mental del conjunto considerado como un todo.

Finalmente, el autor llega a una serie de conclusiones que resumen sus deducciones.

\section{Untrodeduceción}

El año 1956 y para la firma Eiederif Ckel, dedicada a mecánica de precisión, se construyó un edificio de hormigón pretensado, de varias plantas, notable bajo distintos aspectos. Este trabajo va encaminado al estudio del comportamiento dinámico de la estructura basándose en la experimentación directa.

Como el edificio proyectado tenía $16 \mathrm{~m}$ de anchura, la solución inmediata más adecuada parecía ser sin apoyos intermedios, lo que se tradujo en una ventajosa solución, econćmicamente considerable, tratándose de una estructura de hormigón pretensado. Dada la precisión que necesitaban las piezas prefabricadas, el problema oscilatorio presentaba especial interés. Para proceder con rigor se creyó necesario introducir un dispositivo que permitiese modificar voluntariamente el estado vibrante del edificio actuando sobre la frecuencia inducida y evitar la resonancia.

Con objeto de lograr el efecto restrictivo en el sistema vibrante se adoptó una serie de montantes independientes, rígidos y resistentes que pudieran ser montados y desmontados con facilidad. Esios montantes se extenderían desde la planta baja a la superior, siguiendo la vertical y apoyándose en la parte superior en el centro de la luz de las distintas vigas de los forjados.

La explotación del edificio durante cinco años consecutivos permite afirmar que la estructura de hormigón pretensado, de varias plantas y sin pilares intermedios, ha venido comportándose sin perturbación notable en el régimen oscilante estudiado previamente en el proyecto. La introducción en este caso de los referidos montantes no ha sido necesaria.

\section{Bases y dispositivos de los emsagos \\ Objeto de los ensayos}

Antes de la puesta en servicio del edificio se hicieron varios ensayos de efectos dinámicos, de los que interesaba especialmente determinar la frecuencia propia, la oscilacićn y el amortiguamienio, datos todos ellos necesarios para comparar los resultados experimentales con los cálculos analíticos. Era necesario también el estudio de la frecuencia propia del edificio, al ser éste modificado por la introducción de montantes que, como se dijo anteriormente, seguirían la vertical que pasa por los puntos medios de las vigas o dinteles.

El programa de ensayos y mediciones de la oscilación, para la que se emplearon procedimientos exclusivamente eléctricos, fue redactado por instituciones de carácter técnico y especializadas.

\section{Len estrinctina}

El entramado está formado a base de pórticos sencillos, superpuestos, de $15,4 \mathrm{~m}$ de luz, espaciados a $6 \mathrm{~m}$. La estructura consta de cinco plantas completas, y la sexta va retranqueada unos $3 \mathrm{~m}$ respecto a la fachada, y se ha cubierto con una estructura de acero. Las vigas o cabezales de los pórticos son de hormigón pretensado, sistema de Dywidag, con 14 cables de $26 \mathrm{~mm}$ sometidos a un esfuerzo por cable de 4.00 t. Las vigas de la planta baja y los dos pilares del sćtano son de hormigón armado ordinario.

Las vigas tienen $85 \mathrm{~cm}$ de canto y una anchura de $60 \mathrm{~cm}$. En la clave, el alma se ve atravesada por los conductos del aire acondicionado. Los pilares de los pórticos tienen $80 \times 60 \mathrm{~cm}$ de sección media. 


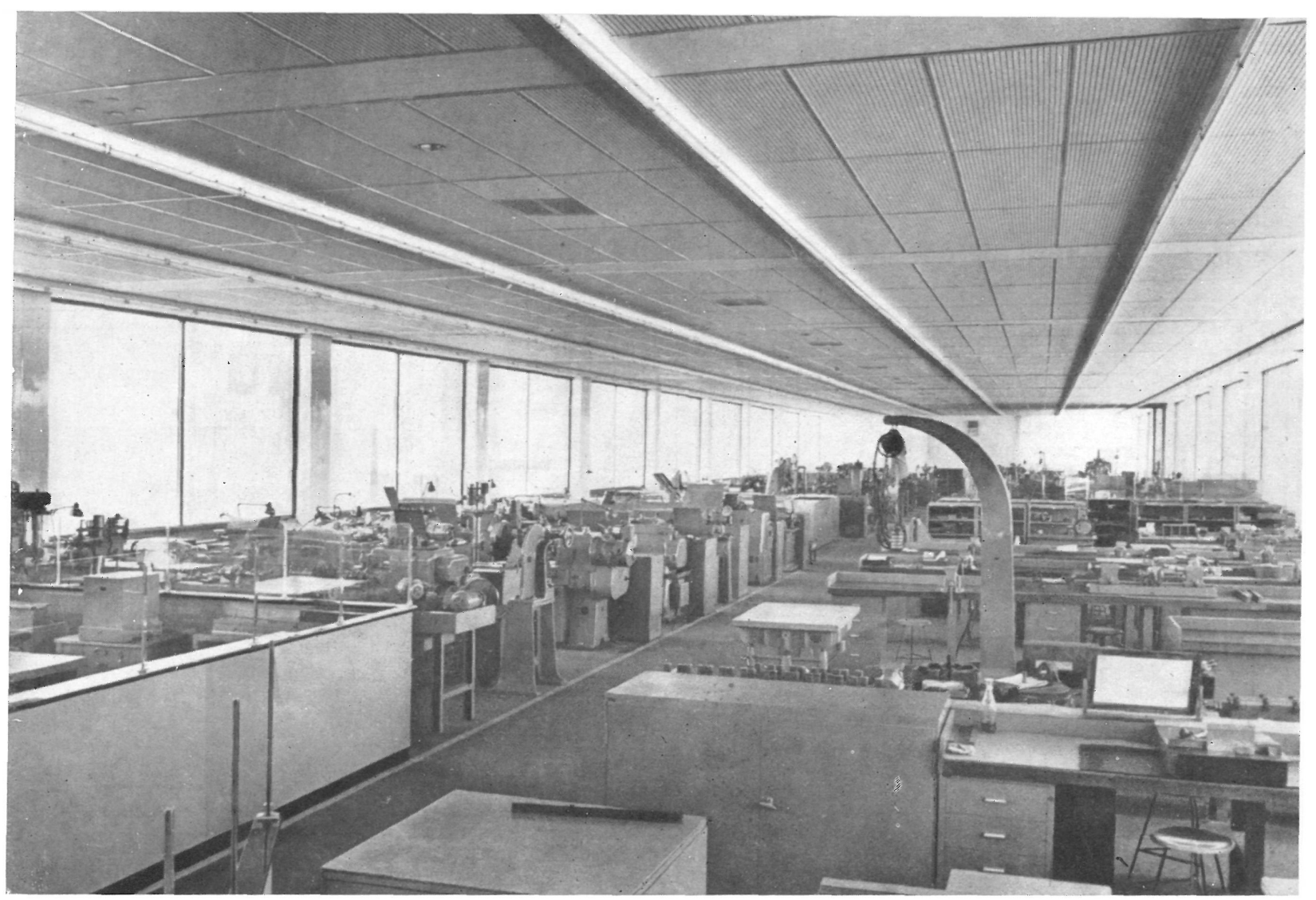

Interior de una de las plantas.

Los forjados están constituidos por un sistema nervado con viguetas de $38 \mathrm{~cm}$ de canto, tesadas en el sentido longitudinal del edificio. En una banda de $3,5 \mathrm{~m}$ de anchura, situada en la parte central del edificio, el forjado está formado por una estructura, tipo cajón, que permite disponer los conductos del aire acondicionado.

La parte ensayada del edificio se halla separada de la torre lateral y entre ambas está situada la junta de dilatación. Las medianerías entre edificio y torre constituyen un tope que puede considerarse rígido. Los montantes independientes empleados para la modificación del sistema vibrante durante los ensayos, consistían en perfiles laminados provistos de dos tirantes compuestos por dos cables de $32 \mathrm{~mm}$ de diámetro. Su comportamiento respecto a la dilatación es similar a la de una columna de hormigón, de $25 \times 25$, armada con cuatro cables de $32 \mathrm{~mm}$. La unión entre la parte superior e inferior de estos montantes se ha verificado por el intermedio de placas de acero recubiertas con yeso, y los tirantes se tesaron con un esfuerzo de $10 \mathrm{t}$ por unidad, con lo cual se consiguieron uniones suficientemente estables.

\section{Oscilladomes}

Las excitaciones periódicas vibrantes se produjeron usando un dispositivo análogo al de las máquinas vibrantes ordinarias, es decir, utilizando dos pesos excéntricamente dispuestos girando en sentidos contrarios y que engendran una fuerza de excitación de determinada dirección, mientras que en el sentido normal a esta dirección se neutralizan las fuerzas centrífugas. La vibradora se unió rígidamente al suelo para producir una excitación de sentido vertical. La parte energética procedía de un motor eléctrico y de mecanismos transmisores sin cambio de marcha.

El momento estático de las masas se midió con mecanismos de palanca, teniendo cuidado de reducir los rozamientos a un mínimo posible. El momento resultante fue de $1.728 \mathrm{~kg} / \mathrm{m}$.

La fuerza centrífuga en la excitadora resultó ser de 6,96 $\mathrm{f}^{2}$. 


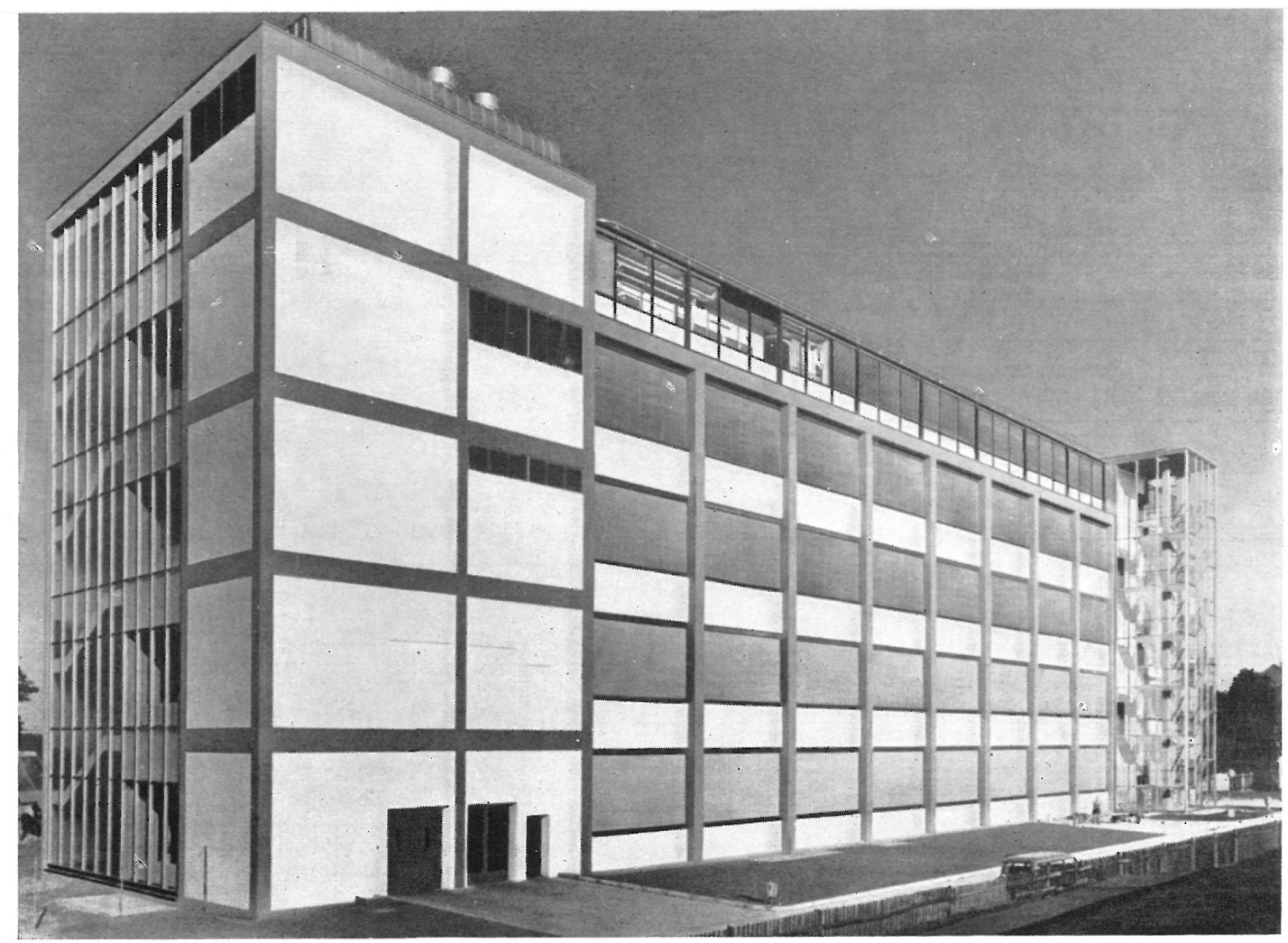

Vista general del edificio.

Para una frecuencia de $20 \mathrm{~Hz}$ (1.200 r.p.m.), la fuerza centrífuga obtenida es de 2.780 kilogramos.

Además se empleó como excitador un torno provisto de un vástago cilíndrico y excéntrico, con objeto de lograr oscilaciones análogas a las que se producen en los procesos de fabricación. El referido vástago tenía $20 \mathrm{~kg}$ de peso y su excentricidad aproximada era de $1 \mathrm{~cm}$, que corresponde a un caso excesivo y poco frecuente en la práctica.

\section{Excitación por Ghoque}

Los choques se produjeron dejando caer una carga desde cierta altura mediante un martinete. La carga era de $1.000 \mathrm{~kg}$ y el peso total de martinete y carga de $2.600 \mathrm{~kg}$. Finalmente, se empleó también un vehículo de transporte de unos $500 \mathrm{~kg}$ de peso para producir los efectos de choque.

\section{Dispositivo de medición}

Las deformaciones periódicas del suelo se midieron con aparatos inductivos. En este sistema los movimientos de la viga originan corrientes inductivas que se refuerzan en un puente y se conducen a un oscilógrafo, que transforma los impulsos eléctricos en movimientos mecánicos y registran la oscilación en una cinta de papel.

Se emplearon dos tipos de emisores inductivos, y en uno de ellos se necesita un punto fijo a partir del cual se mide el movimiento. Este emisor se montó en el piso del entresuelo, pudiéndose admitir que este último es fijo, sin grave error, ya que los dos pilares del sótano le soportan. La deformación de la viga de hormigón pretensado del forjado del entresuelo se transmitía al manipulador del emisor.

El otro emisor inductivo, que trabaja análogamente a un sismógrafo, contiene una masa inerte sostenida con un muelle, y todo ello se halla dentro de una envolvente protectora. La masa queda en reposo al emplear frecuencias suficientemente elevadas. El aparato, colocado directamente sobre la vibradora, recoge las diversas formas de la oscilación. 

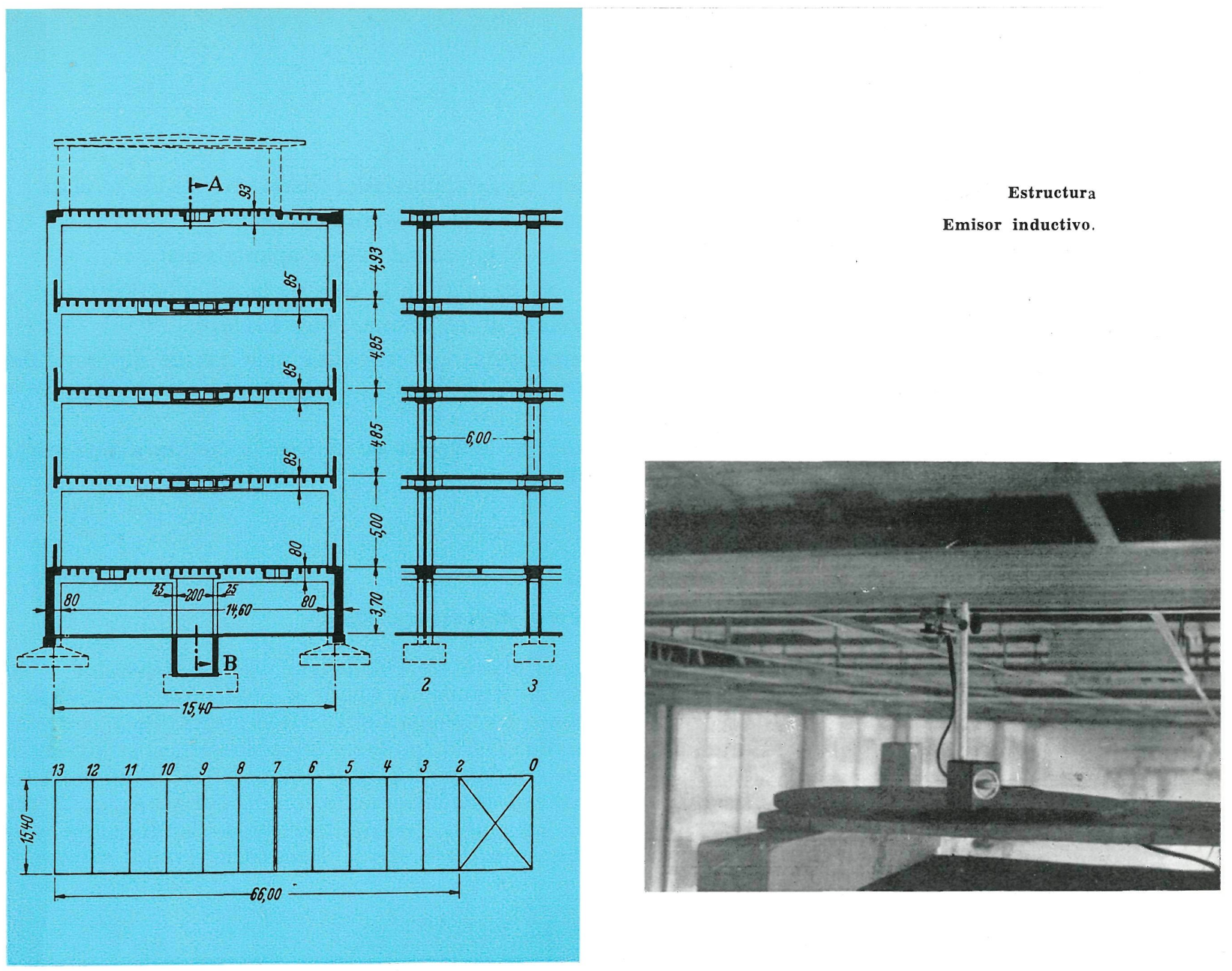

La frecuencia y amplitudes se registran gráficamente en una banda de papel, en la cual puede contarse y medir las oscilaciones, períodos y amplitudes.

\section{Ensagos g representación de las medidas}

Las oscilaciones se producían generalmente en el techo de la planta baja, que es, precisamente, donde se efectuaban las mediciones. Para lograr los diagramas de resonancia se elevaba progresivamente el número de revoluciones de la excitadora, consiguiendo, a los pocos minutos, alcanzar el estado estacionario requerido en cada caso. Partiendo de todo ello, se pudo desarrollar el siguiente programa de ensayos:

a) Fijación de la vibradora en la clave del dintel. Ensayos realizados con la estructura libre, es decir, sin interposición de montantes:

1) Diagrama de resonancia para la clave.

2) Diagrama de resonancia para el punto medio de los pilares.

3) Elástica del pórtico para distintas frecuencias.

4) Propagación de oscilaciones en sentido longitudinal y para las frecuencias características.

b) Fijación del vibrador a un cuarto de la luz de la viga. Ensayo con estructura libre y con montantes intermedios:

1) Diagrama de resonancia para la clave (estructura libre).

2) Diagrama de resonancia para la clave (edificio apuntalado y los dos tirantes colocados en el entresuelo).

3) Diagrama de resonancia para la clave (edificio apuntalado y cables colocados en el entresuelo).

4) Diagrama de resonancia a un cuarto de la luz con el edificio apuntalado. 
5) Diagrama de resonancia en la clave con vigas apuntaladas como en el caso tercero.

6) Elásticas de la viga para distintas frecuencias (edificio apuntalado).

7) Propagación de vibraciones en el sentido longitudinal (vigas apuntaladas).

8) Diagrama de resonancia en la clave con un pilar en la viga de la primera planta.

c) Fijación de un torno en la clave. Diagrama de resonancia para la clave.

d) Amortiguamiento de las oscilaciones producidas por choques (con masas desprendidas de un martinete) y por vehículos de transporte. Excitación y medida en el punto medio de la viga.

e) Determinación de la iínea de influencia de la flecha de la viga sometida a una carga móvil que recorre el eje longitudinal del edificio.

\section{Deducción de los mesultados}

\section{Frecuencias propias y forma de la oscilación. Estructura libre}

Al excitar la viga se producen dos fenómenos claros de resonancia que corresponden a las frecuencias 9,3 y $12,1 \mathrm{~Hz}$. Un tercer fenómeno de resonancia tiende a producirse hacia el final de la zona estudiada para unos $22 \mathrm{~Hz}$. La menor frecuencia de 9,3 Hz corresponde a la oscilacićn fundamental del pórtico total. Dos vigas situadas en pórticos adyacentes vibran con fases opuestas. Como están empotradas elásiicamente en sus apoyos, éstos vibran lateralmente entre los nodos de la estructura. Los pórticos contiguos vibran sincrónicamente con dicha frecuencia. Se trata, por tanto, de un estado plano de vibraciones en que no se producen nodos de vibración en la direccićn longitudinal del edificio. Un cálculo aproximado de la frecuencia propia utilizando la fórmula

$$
f\left[\frac{1}{s}\right]=\frac{5,0}{\sqrt{a \cdot \delta[\mathrm{cm}]}}
$$

dio como resultado una frecuencia propia de $8,2 \mathrm{~Hz}$. La fiecha, $\delta=0,48 \mathrm{~cm}$, se dedujo teniendo en cuenta la dirección variable del peso propio y el efecto horizontal del peso de los pilares, que corresponde a la forma de la oscilación fundamental. El factor de corrección a, menor que 1 , tiene en cuenta la distribución de las masas oscilantes y las condiciones en el apoyo de las vigas. Para vigas simplemente apoyadas y de masa uniformemente distribuidas $a=0,79$, mientras que para vigas con empotramiento completo $a=0,77$, valores determinados para las frecuencias propias, conocidas, de las oscilaciones de flexión. Teniendo en cuenta el empotramiento elástico y la masa del dintel, aproximadamente uniforme, se fijó el valor de 0,78 para $a$. La fórmula aproximada empleada se comprobó utiilizando la de Rayleigh,

$$
f=\frac{1}{2 \pi} \sqrt{\frac{g \cdot \Sigma \mathrm{G} \cdot y}{\Sigma \mathrm{G} \cdot y^{2}}}
$$

que se deduce por consideraciones energéticas, constituyendo una excelente aproximación de la solución exacta. En esta ecuación, $\mathrm{G}$ representa el peso que oscila de un trozo de barra suficientemente corto; $y$ es la flecha estáiica debida al peso $\mathrm{G}$ cuando éste actúa en la dirección de cscilación, y g, la aceleración de la gravedad. El cálculo hecho con esta fórmula da el mismo resultado numérico que el de la ecuación aproximada.

La discrepancia entre la frecuencia propia calculada y la medida es del 12 por 100 y, probablemente, se debe a una estimación por defecto de la rigidez de flexión, es decir, de EJ. Para la calidad del hormigón elegido se supuso que su módulo de elasticidad era de 350.000 kilogramos $/ \mathrm{cm}^{2}$. Puede influir también en la estimación del momento de inercia de la sección de los dinteles, cuyas secciones son muy variables, por lo que la esímación ha de conformarse con ser aproximada.

Con la segunda frecuencia de $12,1 \mathrm{~Hz}$, el pórtico presenta la misma forma de oscilación; sin embargo, el hecho de producirse de nuevo la resonancia se debe a que el estado de oscilación entonces presente es espacial. Los póriticos correspondientes a los ejes numerados $3 / 7$ no 
oscilan ya sincrónicamente; los numerados $3,5,7$, oscilan con fases opuestas, mientras que en los numerados $2,4,6$, aparecen nodos.

En este estado vibrante, la viga longitudinal de acción en forma cajón y, en menor cuantía, el forjado nervado forman elementos que amortiguan las oscilaciones.

El cálculo de este sistema con la ecuación aproximada, comparado con el de oscilaciones sincrónicas de las vigas de hormigón pretensado, dio una mayor frecuencia propia, que va de 8,2 a $15,5 \mathrm{~Hz}$. Teniendo en cuenta lo que anteriormente se dijo sobre la rigidez de flexión, es decir, EJ, la aproximación obtenida con valores medios fue de 9,3-12,1 $\mathrm{Hz}$, respectivamente, que es admitida satisfactoriamente.

La frecuencia propia máxima en la viga, de aproximadamente de $22 \mathrm{~Hz}$, indicada en una de las figuras adjuntas, es también debida a la influencia del estado oscilante que se establece en la dirección longitudinal del edificio. Con esta frecuencia, los pórticos adyacentes numerados 3 a 7 oscilan con fases opuestas. El sistema oscilante puede, por tanto, considerarse como compuesto por una cruceta de la que uno de los elementos está constituido por la viga cajón que tiene $7 \mathrm{~m}$ de longitud. Un cálculo posterior dio una frecuencia de $21 \mathrm{~Hz}$, que coincidió sensiblemente con el valor medio. A la forma simétrica de las oscilaciones de pórtico se superpone otra forma antimétrica. Esto es debido a la posición de la vibradora con excentricidad de un metro respecto al eje del edificio. La frecuencia propia de oscilación del pórtico, completamente antimétrica, resulta mucho mayor analíticamente que el valor obtenido de acuerdo con los resultados de unos 30 hertzios.

Las oscilaciones propias descritas anteriormente se refieren a la explicación directa del eje denominado número 5 . Un resultado análogo se ha obtenido excitando el eje número 6 . Como se esperaba, la frecuencia fundamental de $9,3 \mathrm{~Hz}$ fue mantenida.

La primera oscilación superior se elevó a $16,4 \mathrm{~Hz}$, porque a la resonancia correspondiente de $12,1 \mathrm{~Hz}$ le corresponde un nodo situado en la proximidad inmediata del eje número 6 , pero, sin embargo, esta viga oscila directamente debido al efecto de la vibradora. Un cálculo posterior ha demostrado que la oscilación superior de $16,4 \mathrm{~Hz}$ corresponde a un estado oscilante según la dirección longitudinal del edificio, en el que se producen cuatro nodos en vez de los tres que se pueden apreciar en la curva adjunta correspondiente a la frecuencia 12,1 hertzios.

\section{Frecuencias propias y formas de oscilcakion con In estructura apuntaleda}

Utilizando solamente los dos tirantes de $32 \mathrm{~mm}$ para la unión, las frecuencias y amplitudes propias de las oscilaciones varían poco, debido a la escasa dilatación producida en los tirantes. Unicamente al colocar los montantes laminados aparecen las primeras manifestaciones de las modificaciones. Al solidarizar los forjados del entresuelo y sótano la oscilación fundamental es de $12,8 \mathrm{~Hz}$. La amplitud de oscilación se rebaja considerablemente a pesar del notable aumento de la fuerza centrífuga de la vibradora. La variación, relativamente débil de la oscilación superior de $16,4 \mathrm{~Hz}$ a 17,5 es debida, seguramente, a que, como ocurre también en el edificio libre, se produce un estado oscilante antimétrico para la frecuencia de 21,1 hertzios.

Las medidas obtenidas en la viga adyacente número 5, no apuntalada, dieron también una modificación de la frecuencia fundamental de $12,8 \mathrm{~Hz}$; sin embargo, se nota el efecto de la frecuencia propia de $9,3 \mathrm{~Hz}$. La flecha de estabilidad, con estructura no apuntalada, es para la resonancia $12,8 \mathrm{~Hz}$ casi el doble que la de la viga inmediata apuntalada; por esta razón se propaga a los montantes, por el intermedio de la viga cajón, fuerzas dinámicas intensas que se traducen en una compresión sensible en los apoyos. Solamente si se colocan varios montantes interiores de apoyo tiene lugar la formación de nuevos nodos en la oscilación.

En la figura de la pág. 67 se pueden apreciar los distintos estados de oscilación producidos en la dirección axil del edificio, correspondiente a las frecuencias citadas. Una comparación permite apreciar que la longitud de onda de la oscilación disminuye al aumentar la frecuencia. Esto se hace más sensible en la zona comprendida entre los ejes números 2 y 4. 
En dicha figura se han representado, superpuestas, las oscilaciones correspondientes a la referida zona, en las que se puede reconocer claramente su regularidad. Esto confirma, nuevamente, que las oscilaciones de orden superior medias vienen determinadas por la forma de oscilación en el sentido longitudinal del edificio.

Finalmente, se hizo un ensayo con la primer planta apuntalada como se indica en la figura adjunta, donde se aprecia que en esencia se producen las mismas frecuencias propias que en el edificio sin apuntalar, pero las amplitudes se reducen dos tercios aproximadamente. La frecuencia propia de $9,3 \mathrm{~Hz}$ no ha variado de manera sensible; esto se debe a que se conservan inalterables las formas fundamentales de oscilación en las plantas superiores y en los pórticos adyacentes. Con ello, tanto la perturbación local introducida en la oscilación fundamental al enlazar las dos vigas, como la doble onda originada en el montante de la primera planta, se superponen a la frecuencia propia. Esta perturbación local tiene como consecuencia un ligero aumento de frecuencia en la resonancia, que en ese caso es de 10,3 hertzios.

\section{Efecto do un tormo provisto de vistago exchentrico}

A pesar de la gran magnitud del peso excéntrico, la amplitud máxima en cada caso de resonancia es de sólo $0,1 \mathrm{~mm}$. Este resultado favorable obedece, principalmente, a la gran masa del forjado cuya inercia modera la oscilación, y, en el caso de resonancia, el efecto amortiguador se traduce en trabajo interno de rozamiento.

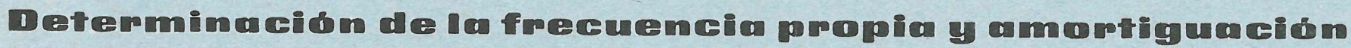 por efectos de choques}

El proceso amortiguador cuando la excitación es debida a percusiones ha sido recogido en un oscilógrafo y motivado por la caída de un peso a partir de cierta altura. Los ensayos se realizaron con un martinete cuyo peso, incluida la carga, era de $2,6 \mathrm{t}$, y se comprobó, contando las oscilaciones, una frecuencia propia de $8,8 \mathrm{~Hz}$. La diferencia de esta frecuencia con la producida con la excitación periódica, que es de $9,3 \mathrm{~Hz}$, se debe, esencialmente, a que la masa vibrante queda incrementada con la del martinete. En los ensayos de choque por movimiento de un vehículo de $500 \mathrm{~kg}$ de peso se obtuvo una frecuencia propia de $9,5 \mathrm{~Hz}$, coincidiendo, admisiblemente, con los ensayos de resonancia.

Una característica del amortiguamiento de la oscilación es el logaritmo del decremento

$$
\delta=\log \frac{y_{n}}{y_{n+1}}=\log y_{n}-\log y_{n+1}
$$
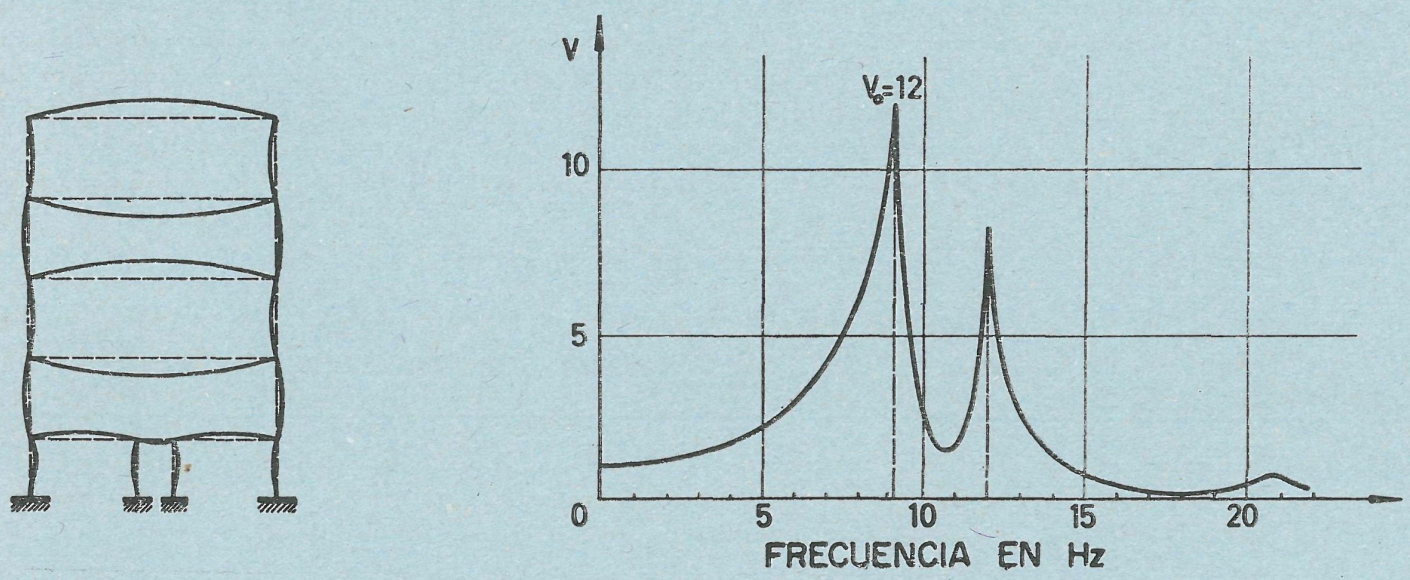

$$
\mathrm{A}=\frac{y_{d y n}}{y_{\text {stat }}}
$$

Oscilación fundamental de la estructura y gráfico del factor dinámico de ampliación. 
válido para un oscilador simple al que se le supone: la fuerza elástica de retroceso en cada instante es proporcional al camino recorrido, y la fuerza de amortiguamiento es proporcional a la velocidad, como corrientemente se admite. Para dos oscilaciones consecutivas $y_{n} / y_{n+1}$ es constante. Para $n$ vibraciones seguidas se tendrá:

$$
\delta=\frac{\log y_{0}-\log y_{n}}{n}
$$

en la que $n$ representa el número de vibraciones.

En caso de pequeñas oscilaciones se cumple la hipótesis relativa a las fuerzas, es decir, proporcionalidad al camino recorrido y velocidad. Sin embargo, no existen vibradores simples formados por una masa oscilante concentrada en un punto. A esto se debe, en muchos casos, que el amortiguamiento de las oscilaciones discrepe sensiblemente. Se ve, por los diagramas adjuntos, que al proceso de amortiguamiento. se superponen otras ondas debidas a reflexiones internas o choques locales dentro del sistema. La ecuación anterior se utiliza para el cálculo de decremento y se aplica a la fase inicial del proceso, durante el cual se supone que no se ha producido fenómeno alguno de reflexión. De nueve procesos de amortiguamiento ensayados se obtuvieron los valores siguientes:

$\begin{array}{crrrc}\mathrm{N}_{r} & y_{0} & y_{n} & n & \delta \\ 1 & 12,5 & 3,2 & 6 & 0,228 \\ 2 & 10,5 & 2,0 & 6 & 0,270 \\ 3 & 11,1 & 4,0 & 5 & 0,208 \\ 4 & 9,1 & 2,0 & 5 & 0,300 \\ 5 & 9,0 & 2,1 & 4 & 0,350 \\ 6 & 17,0 & 6,9 & 4 & 0,380 \\ 7 & 7,5 & 3,6 & 2 & 0,240 \\ 8 & 10,1 & 5,0 & 2 & 0,230 \\ 9 & 7,0 & 3,0 & 2 & 0,280\end{array}$

El valor medio del decremento logarítmico es de 0,275.

El llamado coeficiente de amortiguamiento es:

$$
\mathrm{D}=\frac{\delta}{2 \pi}=0,044
$$

Conocido el amortiguamiento queda también determinado el llamado factor dinámico de ampliación o relación de la flecha dinámica a la estática o, también, de la oscilación dinámica a la estática. Para el factor dinámico de ampliación:

$$
V=\frac{1}{\sqrt{\left(1-\eta^{2}\right)^{2}+4 D^{2} \cdot \eta^{2}}}
$$

Donde $\eta=f / f_{1}$ es la relación entre las frecuencias de excitación y la propia. Cuando es bajo el coeficiente $D$ de amortiguamiento, como sucede en los materiales, se tienen, aproximadamente, $\mathrm{V}=1 /\left(1-\eta^{2}\right)$, siempre que $\eta$ no sea próximo a la unidad. De aquí se deduce que en la zona fuera de la resonancia, el factor de ampliación es prácticamente independiente del amortiguamiento.

En el caso de resonancia, es decir, para $\eta=1$, se deduce, por el contrario, según la ecuación anterior, que el factor de ampliación $\mathrm{V}_{1}$ (llamado por Rausch coeficiente de resonancia), depende solamente del coeficiente de amortiguamiento, es decir,

$$
\mathrm{V}_{1}=\frac{1}{2 \mathrm{D}}=\frac{\pi}{\alpha} \text { que para } \mathrm{D}=0,044 \text { se tiene: } \mathrm{V}_{1}=11,4
$$




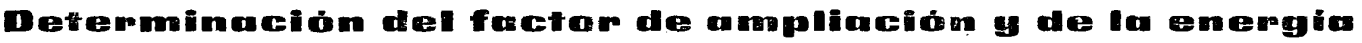

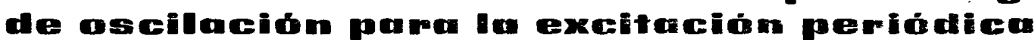

Como se ha indicado anteriormente, el coeficiente de resonancia puede también determinarse como relación entre las flechas dinámica y estática en el caso de resonancia o, lo que es lo mismo,

$$
\mathrm{V}_{1}=\frac{y_{\text {din }}}{y_{\text {st }}}
$$

La flecha dinámica admitida para el caso de excitación en el punto medio de la viga da $9,3 \mathrm{~Hz}, \mathrm{y}$ :

$$
y_{d i *} \frac{830}{2}=4,15 \mu
$$

La fuerza centrífuga de la vibradora es $p=6,96 f^{2}=6,96 \times 9,3^{2}=600 \mathrm{~kg}$.

La flecha estática, tomada en la línea de influencia de la carga para sobrecarga estática, da el valor

$$
y_{\text {. st }}=93 \cdot \frac{600}{1.300}=34 \mu
$$

El factor dinámico de ampliación en el caso de resonancia es $\mathrm{V}_{1}=12,2$.

De acuerdo con la ecuación

$$
\mathrm{V}_{1}=\frac{1}{2 \mathrm{D}}=\frac{\pi}{\delta}
$$

el amortiguamiento es $\mathrm{D}=0,041$; el decremento, $\delta=0,258$.

La coincidencia con los valores determinados de ensayos de choque $\left(V_{1}=11,4 ; D=0,044\right.$; $\delta=0,275$ ) es satisfactoria. Por término medio puede admitirse: $V_{1}=12 ; D=0,042 ; \delta=0,26$.

Se puede admitir en toda la zona de frecuencias la ley del factor de ampliación: $\mathrm{V}=y_{d i n}$ l $/ y_{\text {est }}$. Como la flecha estática crece con la fuerza centrífuga de la vibradora a razón del cuadrado del número de revoluciones, se obtiene la ley de variación del factor de ampliación en función de la frecuencia de la excitación reduciendo las amplitudes (flechas) medidas con el factor $c / f^{2}$. El factor $\mathrm{C}$ se determina teniendo en cuenta que para la resonancia $9,3 \mathrm{~Hz}$ se ha hallado ya el factor de ampliación $V_{1}=12$. El diagrama de resonancia que así se obtiene queda referido a una fuerza de excitación constante e independiente de la frecuencia.

Conociendo ya el coeficiente de resonancia $V_{1}=12$ y las amplitudes (flechas) medidas, podemos hallar el peso del vástago excéntrico que produce la excitación cuando gira en un torno. A la amplitud de $50 \mu$ corresponde, exceptuando el debido cálculo, una fuerza dinámica de: $\mathrm{P}_{d i n}=790 \mathrm{~kg}$. La fuerza centrífuga efectiva $\mathrm{P}$ vale, pues: $\mathrm{P}=p_{\text {din }} / \mathrm{V}_{1}=790 / 12,2=65 \mathrm{~kg}$. Igualando esta fuerza centrífuga a m.r. $\mathrm{w}^{2}$ y transformando convenientemente esta ecuación, se tiene, para el momento estático del vástago, $\mathrm{M}=19 \mathrm{~cm} / \mathrm{kg}$, lo que corresponde a una barra redonda de hierro de $19 \mathrm{~kg}$ de peso y $1 \mathrm{~cm}$ de excentricidad.

Una magnitud notable es la cantidad de energía absorbida por el amortiguamiento en la unidad del tiempo y, respectivamente, ia eneraía de oscilación suministrada por la vibradora en el caso de resonancia. 
Si la excitación es periódica, la fuerza excitadora y la amplitud están en diferencia de fase exactamente igual a un cuarto de período. Esto nos permite determinar del siguiente modo el trabajo realizado por la fuerza de excitación para que la viga efectúe una oscilación de flecha $y$. Con ello tendremos:

$$
\begin{gathered}
\mathrm{A}_{1}=\int_{0}^{\mathrm{T}} \mathrm{P} \cdot d y=\int_{0}^{\mathrm{T}}\left(\mathrm{P}_{0} \cdot \cos \omega t\right) \cdot\left(y_{0} \cdot \omega \cdot \cos \omega t\right) d t= \\
=\mathrm{P}_{0} \cdot y_{0} \int_{0}^{\mathrm{T}} \cos ^{2} \omega t \cdot d t=\mathrm{P}_{0} \cdot y_{0} \cdot \pi \cdot
\end{gathered}
$$

La energía consumida en la unidad de tiempo es:

$$
\begin{gathered}
\mathrm{L}=\frac{\mathrm{A}_{1}}{\mathrm{~T}}=\mathrm{A}_{1} \cdot f=\pi \cdot \mathrm{P}_{o} \cdot y_{0} \cdot f=3,14 \cdot 600 \cdot 415 \\
\cdot 10^{-6} \cdot 9,3=7,3 \mathrm{~m} \cdot \mathrm{kg} / \mathrm{s}=0,097 \mathrm{CV} .
\end{gathered}
$$

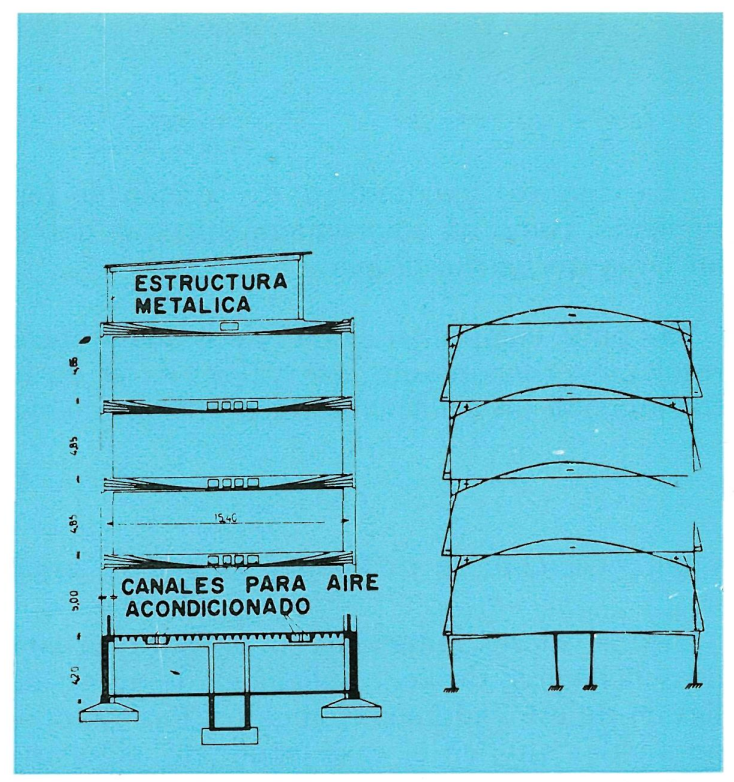

Esquemas de momentos y de vigas pretensadas.

En este cálculo se supone que la fuerza centrífuga, $\mathrm{P}=6,96 f^{2}$, no queda influida por las amplitudes, lo cual es bastante aproximado.

Se deduce de todo esto que la vibradora, aun en el caso de resonancia, transmite al sistema oscilante una potencia relativamente pequeña. La mejor precaución para evitar oscilaciones sería la degradación de esa energía por medio de amortiguadores adecuados; sin embargo, este método fracasa por los pequeños recorridos (amplitud de oscilación) que han de realizarse en dicha degradación.

\section{Resiumen}

De los ensayos realizados con excitaciones periódicas y de choque, se dedujo, para la frecuencia propia fundamental de la estructura estudiada, el valor de 9,3 Hz. También se hallaron frecuencias de grado superior: $12,1 \mathrm{~Hz}, 16,4 \mathrm{~Hz}, 22 \mathrm{~Hz}$, para casos de resonancia. Conviene notar que, en todos los casos, la forma de oscilación en pórticos aislados es igual a la fundamental; no obstante, se producen en el sentido longitudinal de la estructura otras formas de oscilación.

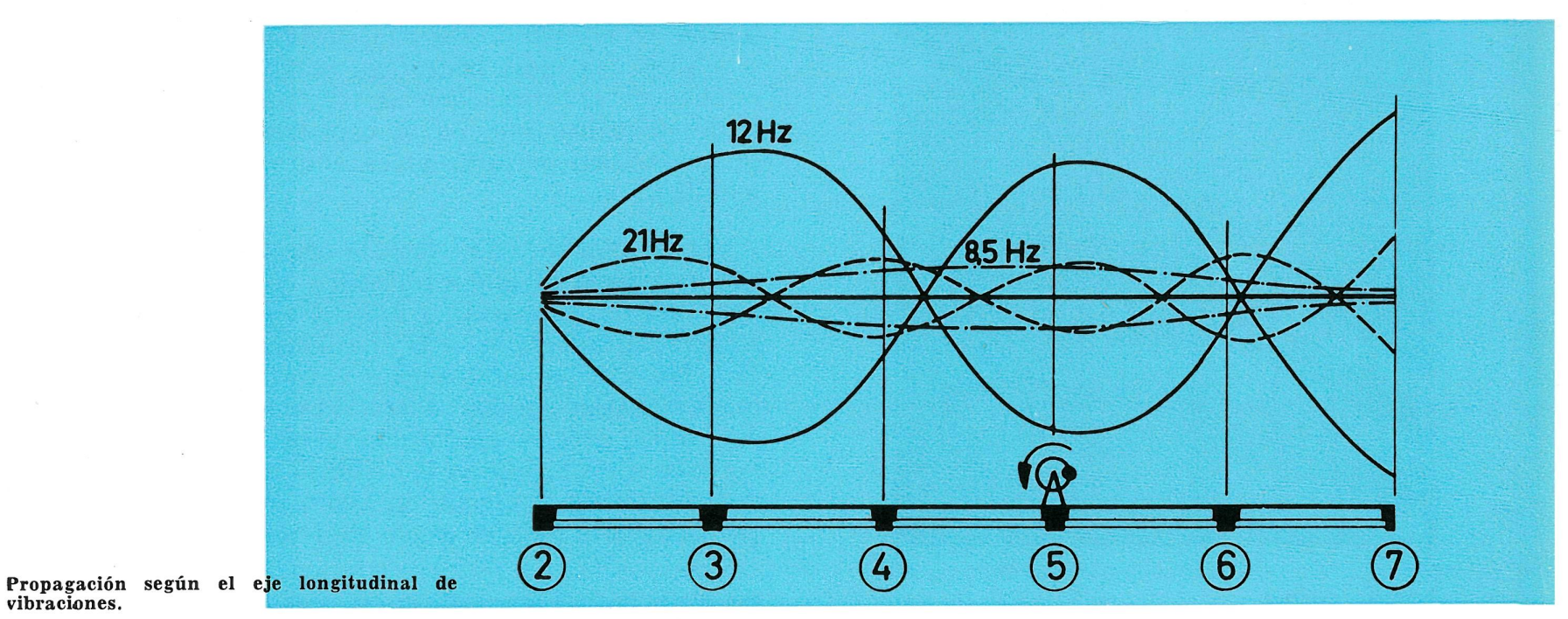


La viga axil longitudinal, de sección de forma cajón, tiene bastante rigidez a la flexión y actúa, por consiguiente, como un amortiguamiento que debilita las oscilaciones de las masas e influye, por tanto, en el valor de la frecuencia propia.

Se pudo comprobar que el decremento logarítmico como medida del amortiguamiento es de $\delta=0,26$, resultando concordante este valor con el de los ensayos de excitación periódica y de choque. A este valor corresponde un coeficiente de amortiguamiento:

$$
\mathrm{D}=\frac{\delta}{2 \pi}=0,042
$$

y un coeficiente de resonancia (factor de ampliación en caso de resonancia) $V_{1}=1 / 2 \mathrm{D}=12$.

Estos valores numéricos no constituyen constantes del material, ya que dependen, además, de la clase de solicitación, de las condiciones de apoyo de las vigas y, en última instancia, de la forma variable de la flexión de estos últimos elementos. Por tanto, no pueden aplicarse a otras construcciones. Además, es preciso no olvidar que, en el caso estudiado, algunas partes importantes de la estructura, tales como los forjados nervados, la viga longitudinal de sección en forma cajón y los pilares son de hormigón armado ordinario sin tensado alguno anterior o posterior.

En los ensayos realizados, el decremento, $\delta=0,26$, corresponde a un amortiguamiento equivalente al de una estructura de hormigón armado y pretensado. En algunas construcciones de hormigón armado se han hallado valores para $\delta$ comprendidos entre 0,28 y 0,60 . En un puente de hormigón pretensado dicho valor era de 0,16. Recordaremos que en construcciones mixtas, es decir, de hormigón y metálica, se han obtenido valores del orden $0,4, y$ en una, exclusivamente metálica, se halló el valor de 0,09 para $\delta$.

Estos valores se resumen en el cuadro siguiente:

\begin{tabular}{|c|c|c|c|}
\hline \multirow{2}{*}{$y_{n /} y_{n_{+1}}$} & $\grave{\partial}=\log y_{n}$ & \multirow[b]{2}{*}{ 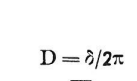 } & \multirow[b]{2}{*}{$\mathrm{V}_{1}=1 / 2 \mathrm{D}$} \\
\hline & $=\log y_{n_{+1}}$ & & \\
\hline $1,32+1,82$ & $0,28+0,60$ & $0,045+0,095$ & $11,0+5,3$ \\
\hline 1,30 & 0,26 & 0,042 & 12 \\
\hline 1,17 & 0,16 & 0,025 & 20 \\
\hline 1,44 & 0,40 & 0,064 & 8 \\
\hline 1,09 & 0,07 & 0,014 & 36 \\
\hline
\end{tabular}

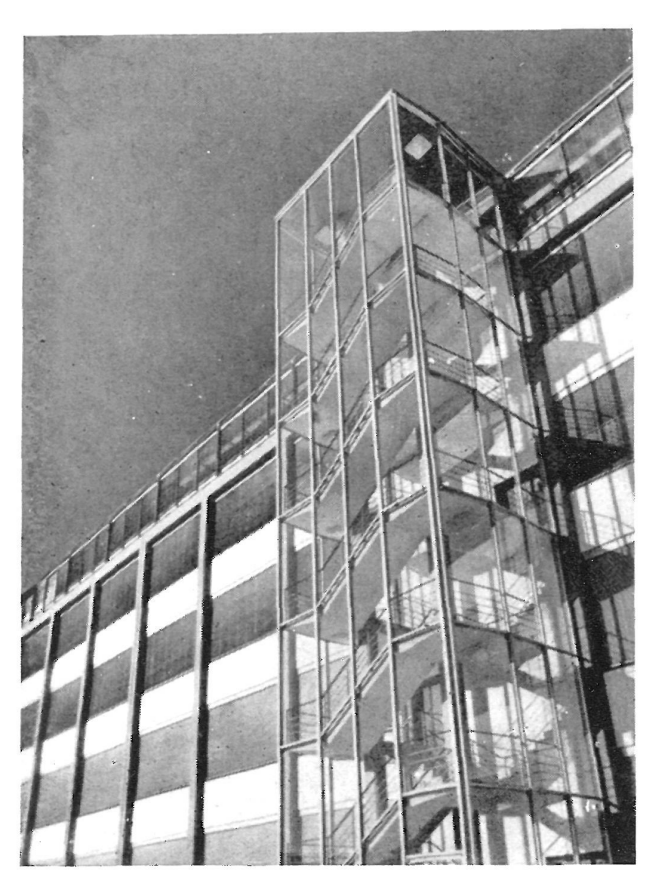

Escalera exterior.

Construcciones de armado $\ldots \ldots \ldots$ Construcción estudiada en este artículo $\ldots$ Puente de hormigón pretensado $\ldots . . . . .$. Construcción compuesta (mixta) .........

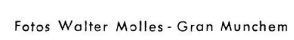

Construcción de acero. Donde: $y_{n} / y_{n+1}$ es la relación de amplitudes de la oscilación libre; $\delta$, el decremento logarítmico; $\mathrm{D}$, el coeficiente de amortiguamiento, y $\mathrm{V}_{1}$, el factor de ampliación en la resonancia, es decir, el coeficiente de esta última.

Las máximas amplitudes de los ensayos con el torno fueron, en el caso de resonancia, de $\pm 0,05 \mathrm{~mm}$, aunque se utilizó una masa centrífuga exagerada con momento estático de aproximadamente $0,2 \mathrm{~cm} / \mathrm{kg}$. Con todo ello pudo deducirse que en la explotación del edificio no se producirían perturbaciones, cosa que ha sucedido en realidad.

El apuntalado entre forjados de sótano y entresuelo, con un pilar resistente a tracción y compresión, redujo la amplitud de resonancia a $2 / 3$ del valor que tiene en el edificio sin apuntalar, y la frecuencia se elevó de 9,3 a $12,8 \mathrm{~Hz}$ y el apuntalamiento entre forjados en la primera planta no produjo alteración medible de la frecuencia propia, pero las amplitudes de oscilación se redujeron también a los 2/3 de la del edificio libre. De ello se deduce que basta intercalar un solo montante para eliminar prácticamente todo el efecto de resonancia.

Información obtenida de la revista "Der Bauningenieur»n. ${ }^{\circ} 3$-1962 


\title{
L'oscillation dans les stuructures en böton proḱcontraint d'étrag ges multiples
}

H. Kupfer, Dr. ingénieur.

Le projet pour la construction d'un édifice industriel destiné à la fabrication de mécanismes de précision a été réalisé en tenant

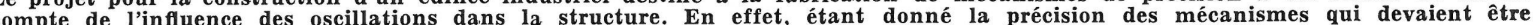
fabriqués, ces effets présentaient un problème d'importance notable. Tout le dispositif structural a été résolu en accord avec ces directrices et de telle façon que l'on puisse comparer les prévisions analytiques avec des essais obtenus directement dans l'édifice même.

L'édifice mesure $66 \mathrm{~m}$ sur 15,4 de surface et comporte: sous-sol, entresol, quatre étages normaux, plus un étage en retrait d'environ ( briquées, on dut veiller d'une manière toute spéciale à leur finissage, car le système vibrant de l'ensemble se voit hautement influencé par ces pièces. Etant donné la grande longueur de cet édifice et son peu de largeur relative, on pensa depuis le début à une solution à base de portiques, de $15 \mathrm{~m}$ de portée, avec des montants de béton armé et des poutres de béton précontraint. La partie centrale des planchers pour les divers étages est constituée par un élément de section creuse, utilisé pour loger les conduits d'air conditionné.

Comme les $15 \mathrm{~m}$ de portée des poutres précontraintes pouvaient avoir une influence notable sur le système vibrant, on a prévu ne série de montants, indépendants, qui pourraient le monter et démonter facilement et qui,

Dans le programme d'essais on tint compte des diverses hypothèses et dispositifs d'excitation ondulatoire et dispositif de régistre par oscillographe.

L'auteur s'étend, avec beaucoup de détails, sur divers essais réalisés et sur les méthodes utilisées pour recueillir les données qui permettraient l'analyse générale du système vibrant, ses modifications générales et résonances, amplitudes et onde propre selon
les divers cas expérimentés, le tout devant servir de base pour arriver au résultat final, c'est-à-dire arriver à la fréquence fondamentale de l'ensemble considéré comme un tout.

Finalement, l'auteur arrive à une série de conclusions qui résument ses déductions.

\section{Vibration in Multistorey Prestressed Concrete Structures}

H. Kupfer, doctor engineering.

The design of an industrial building intended for the manufacture of precision instruments was carried out taking account of the influence of small vibrations of the structure. Owing to the detailed accuracy of the instruments to be manufactured, this became an structure was so planned and constructed that the analical estimates of its dynamic performance could be checked a posteriori by means of direct tests on the building.

The building has a ground area of 66 by $15.4 \mathrm{~ms}$ and consists of basement, semibasement, four normal floor levels and a top storey, set back about $3 \mathrm{~ms}$ from the main frontage. The roof is in metal. As the building has been mainly done with prefabricated units, it was especially important that these should be accurately finished, since the dynamic stability (vibrations) of the structure depended very largely on the prefabricated units. Owing to the large length to width ratio of the building, a design based on the use of portal frames was adopted from the outset. These frames have a $15 \mathrm{~ms}$ span, the vertical members being reinforced concrete, and the horizontal beams are prestressed concrete. The central part of the flooring structure, at the various floor levels, consists of a hollow nuit, which carries the air conditioning ducts.

As the $15 \mathrm{~ms}$ span of the beams could influence considerably the mode of vibration of the structure, provision was made to fit a number of additional struts. They would be easy to put in position and remove, and placed at the midspan of the horizonta beams, would supply a support that would influence notably the overall behaviour of the building structure.

The initial test program sought to cover various behaviour hypotheses and systems of induced oscillations. A number of arrangements of the recording oscillographs were also tried.

The author of this article describes in considerable detail the various tests and the methods of obtaining the necessary experimental data to analyse the dynamics of the system, its modifications and the natural frequency, harmonics and amplitudes corresponding considered as a whole. Finally the author draws a number of general inferences from the investigation.

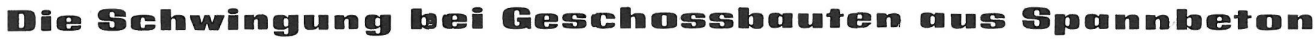

\author{
H. Kupfer, Dr. Ingenieur.
}

Das Projekt für den Bau eines für die Herstellung von Präzisionsgeräten bestimmten Industriegebäudes wurde ausgeführt, indem man den Einfluss der Schwingungen auf die Struktur in Betracht zog, eine Tatsache, die wegen der Genauigkeit der herzustellenden Geräte ein Problem von beachtenswerter Bedeutung darstellte. Die ganze strukturelle Vorrichtung wurde im Einklang mit diesen Richtlinien gelöst und derart, dass sich die analytisch Voraussichten mit den direkten, am eigentlichen Gebäude erhaltenen

Das Gebäude weist im Grundriss $66 \times 15,4 \mathrm{~m}$ auf und besteht aus einem Keller, einem Erdgeschoss, vier normalen Stockwerken, einem sechsten Geschoss, das hinsichtlich der Fassade um etwa $3 \mathrm{~m}$ zurückgesetzt ist, und einem Dach aus Stahlgefüge. Da für die Errichtung des Baues vorgefertigte Stücke verwendet wurden, musste man bei der Herstellung derselben eine besondere Vorsicht beobachten, da das Schwingungssystem des Ganzen durch diese Stücke stark beeinflusst wird. Wegen der grossen Länge des Gebäudes und seiner verhältnismässig geringen Breite dachte man von vornherein an eine Lösung auf Grund von Rahmen mit $15 \mathrm{~m}$ Weite, mit Pfosten aus Stahlbeton und Balken aus Spannbeton. Der Mittelteil der Decken in den verschiedenen Stockwerken wird von einem im Querschnitt hohlen Elemente gebildet, das für die Unterbringung der Klimatisierungs-Leitungen

Da die Weite der Spannbalken von $15 \mathrm{~m}$ auf das Schwingungssystem einen bemerkenswerten Einfluss ausüben könnte, wurden eine Reihe von unabhängigen Pfosten vorgesehen, die sich leicht anbringen und abbauen liessen, und die eine Art allgemeiner Abstützung von beachtenswertem Einfluss besorgen würden, wenn sie in die Mitte eines jeden Rahmens gestellt werden.

Im Versuchsprogramm zog man verschiedene Voraussetzungen und Vorrichtungen von wellenförmigen Erregungen und Aufzeichnungs-Vorrichtungen an Oszillographen in Betracht.

Der Verfasser dehnt sich in aller Einzelheit in den verschiedenen ausgeführten Versuchen und in den benützten Methoden aus, um die Daten zusammenzufassen, die eine allgemeine Analyse des Schwingungssystemes, seine allgemeinen Veränderungen, seinen Nachhall, die Schwingungsweiten und eigenwe, dis zu erreichen. Schliesslich gelangt der Verfasser zu einer Reihe von Schlussfolgerungen, die seine Ableitungen zusammenfassen. 